\title{
Simulation of unconventional well tests with the finite volume method
}

\author{
CANCELLIERE Michel and VERGA Francesca*
}

Politecnico di Torino, DITAG, Torino (TO) 10129, Italy

(C) China University of Petroleum (Beijing) and Springer-Verlag Berlin Heidelberg 2012

\begin{abstract}
The finite volume method has been successfully applied in several engineering fields and has shown outstanding performance in fluid dynamics simulation. In this paper, the general framework for the simulation of near-wellbore systems using the finite volume method is described. The mathematical model and the numerical model developed by the authors are presented and discussed. A radial geometry in the vertical plane was implemented so as to thoroughly describe near-wellbore phenomena. The model was then used to simulate injection tests in an oil reservoir through a horizontal well and proved very powerful to correctly reproduce the transient pressure behavior. The reason for this is the robustness of the method, which is independent of the gridding options because the discretization is performed in the physical space. The model is able to describe the phenomena taking place in the reservoir even in complex situations, i.e. in the presence of heterogeneities and permeability barriers, demonstrating the flexibility of the finite volume method when simulating non-conventional tests. The results are presented in comparison with those obtained with the finite difference numerical approach and with analytical methods, if possible.
\end{abstract}

Key words: Finite volume method, numerical methods, horizontal well modeling, injection testing, gridding

\section{Introduction}

Nowadays, the use of computers and numerical models is a standard in almost all sectors of the oil industry. It would have been impossible to develop the technologies currently used to exploit hydrocarbon reservoirs without computers and, as a consequence, to reach today's production and recovery levels. Even if numerical models and computational techniques are not exclusive to the oil industry, a symbiotic relation has been established between them to such a point that several breakthrough technologies in computational science have come from the oil industry. At the same time, the oil industry has benefited from the developments in the computational field achieved in other industrial sectors.

In this context, reservoir simulation has been one of the technologies that has improved the most. By the mid1950s, reservoir simulators subtly started to be part of reservoir studies (Mattax and Dalton, 1990). In the following decades, they gained popularity in the industry and currently represent an essential tool that no petroleum engineer can do without. At present, simulators are quite different from their predecessors of the past century: in 2011 a single desktop computer can perform orders of magnitude more operations per second than one of the large mainframe computers

*Corresponding author. email: francesca.verga@polito.it Received October 11, 2011 available in 1955. Furthermore, the decrease in cost of computational power and recent developments in parallel architectures have brought reservoir modeling to new levels.

Because of the improvements in the technologies for formation characterization, well drilling and reservoir monitoring, the trend in static and dynamic simulation continuously needs more accurate modeling with higher levels of detail. These technological improvements provide the possibility to capture all the reservoir geological and geomechanical features and the ability to quickly and accurately describe complex interactions among rocks, produced/injected and resident fluids, and wells. However, a balance between accuracy and speed of computation is necessary for detailed models. This is even more so in the case of the application of meta-heuristics approaches for history matching and forecasting, where the large number of simulations required can lead to a very time consuming process that, in the case of very large reservoir models, may even not be feasible.

Traditionally, numerical models based on the finite differences method (FDM) were developed for the discretization of the partial differential equations (PDEs) governing multi-phase flow in porous media; finite element methods are generally not well suited to describe the fluid flow because they might not guarantee the conservation of mass (Faust and Mercer, 1976; Wan et al, 2003). In recent years, new methods have been tested in order to obtain better accuracy by using more complex grids that adapt to 
the flow patterns as determined by the reservoir geological features and by the well's architecture and pattern. Among these methods, the finite volume method (FVM) appears to be extremely promising. Even though it has not yet been extensively adopted in reservoir simulators, the finite volume method has proven to work well in complex geometries and is already used in commercial fluid-dynamic simulators. In this paper, the FVM is documented and successfully applied to the simulation of near-wellbore two-phase flow occurring in unconventional well testing.

\section{The finite volume method}

The FVM is a discretization technique which is well suited for numerical simulations of various types (i.e., elliptic, parabolic or hyperbolic) of conservation laws. It has been used in several engineering fields, such as fluid mechanics, heat and mass transfer as well as petroleum engineering (Eymard et al, 1997). In the context of hydrocarbon reservoir simulation, the finite volume method was applied for the first time by Lemonnier (1979).

The FVM, similar to other methods such as the FDM and the finite element method, provides the representation and evaluation of PDEs when expressed in the form of algebraic equations. The variables or parameter values are calculated in small volumes surrounding each cell node. These small volumes are known as the "finite volume". The surface integrals are approximated by the sum of the fluxes through each of the sides of the control volume. To fully appreciate the advantages of the FVM, it is important to recall that spatial discretization is carried out directly in the physical space. As a consequence, there is no need for shifting between coordinate systems as in the finite difference method (Blazek 2001). Thus, FVM is a very flexible method that can be easily applied to structured and unstructured grids. For this reason, the finite volume method is commonly used in problems involving flows in complex geometries. The finite difference methods are locally conservative, but they are not robust enough to deal with complex domain geometries. On the other hand, since the FVM is based on the direct discretization of the conservation laws, the mass is conserved by the numerical scheme. This condition makes the FVM very attractive relative to the other methods (Blazek, 2001). It is also important to point out that in some cases the FVM can be equivalent to the FDM or to a low-order finite element method; under these circumstances, the two methods can be validated against each other.

The FVM is not a new idea and it has already been applied in reservoir simulation. In fact, the finite volume method was initially applied by Rozonin (1989) for the simulation of single-phase flow in order to overcome the problem of mass conservation related to the classical Galerkin or variational finite element methods. Later, Santos et al (1992) efficiently simulated miscible displacement in two-dimensional reservoirs using a finite-volume approach that included a nine-point discretization scheme based on the flux interpolation technique. The authors highlighted the effectiveness of the FVM in reducing numerical dispersion and grid orientation effects for adverse mobility ratios. In 1994, Amado and Pedrosa (1994) applied the finite volume method to describe a $2 \mathrm{D}$ complex reservoir geometry with arbitrary irregular boundaries by using a triangular mesh. The results showed that the finite volume approach led to an accurate representation of the flow in the reservoir. Dickstein et al (1997) presented a finite volume model for the simulation of single-phase flow of a slightly compressible fluid in a reservoir drained by a horizontal well. The grid was locally refined around the well to efficiently handle different time scales in a robust way. Recently, the multi-scale finite volume method was used by Hajibeygi et al (2011) to effectively deal with complex faulted reservoirs; following the approach suggested by the authors, accurate quantities at the coarse scale can be calculated by using basis and correction functions computed in the extended local domain near the fault region. Mishev and Jiang (2011) successfully applied a mixed multi-scale FVM approach to solve reservoir simulation problems with strong local heterogeneities. The method offers the flexibility to use the multi-scale space for the calculation of the pressure and/or velocity fields, yet global information can be incorporated if necessary.

In this paper, an original contribution for the application of the FVM is provided. A new application, which is particularly useful in the field of unconventional well testing, is presented and discussed. The method is particularly suited for the simulation of multi-phase near-wellbore fluid flow.

\section{Unconventional well testing}

Well testing, which consists in producing hydrocarbon to the surface while measuring the pressure variations induced in the reservoir, has been used for decades for reservoir appraisal. However, due to more stringent environmental regulations and a general need for reduced operating expenses, alternative test procedures are often adopted, especially in exploration wells, in place of the typical production/ build-up sequence. This is particularly true in deep-water and arctic environments where conventional tests can be prohibitively expensive or logistically unfeasible (Soliman et al, 2004; 2005) as well as in several protected areas of the world where no emissions are allowed to be released into the environment. One of the most interesting new unconventional well testing methodologies is injection testing. Injection tests eliminate emissions during reservoir appraisal and, except for fluid sampling, can provide all the information needed to estimate the well productivity at a reasonably low cost with a good degree of reliability (Levitan, 2002; Beretta et al, 2007). Injectivity tests have been performed for a long time. However, they are not the same as injection tests. Injectivity tests have the target of measuring the amount of water that can either be injected into an oil reservoir for water flooding purposes or be disposed into an underground layer. For this reason they did not involve any sort of pressure transient interpretation. An injection test consists of injecting a fluid, typically brine, diesel or nitrogen, in a potential pay zone (Verga and Rocca, 2010). During the injection period and the subsequent fall-off period, in which the well is shut-in and the 
pressure declines to the initial equilibrium value, the reservoir pressure response is monitored (Fig. 1).

This methodology increases the difficulties in well test interpretation because of the presence of two mobile phases in the reservoir: the fluid originally in place (hydrocarbon) and the injected fluid (Gunawan et al, 2002; Verga et al, 2008). The fluid saturations and the permeability of the reservoir rock to each fluid change dynamically during injection both in space and time. Only very favorable mobility ratios induce displacement that can be described by a piston-like model (mobility is the ratio between effective permeability and fluid viscosity; the ratio between the mobility of the displacing fluid and that of the displaced fluid is the mobility ratio).

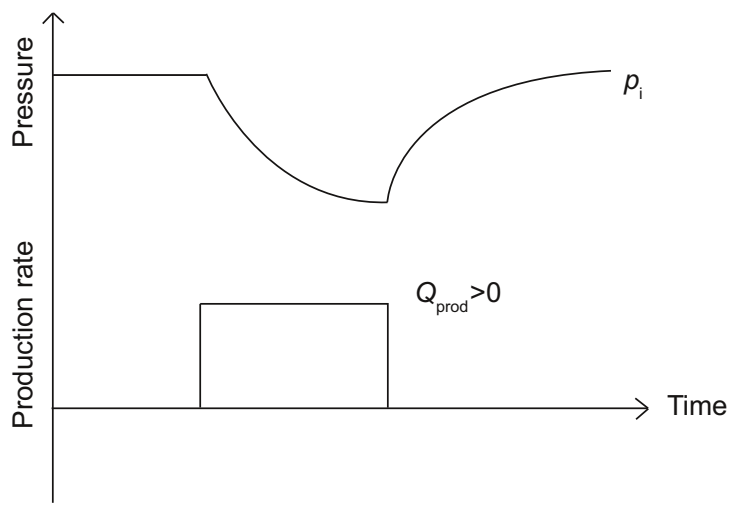

(a)
Moreover, gravitational and thermal gradients, heterogeneity and anisotropy might strongly affect the fluid distribution, resulting in an inadequacy of analytical models to reproduce the pressure transient behavior. On the other hand, the use of a numerical model associated to an adequate gridding offers the capacity to account for all the relevant physical phenomena taking place in the porous media. This even holds true for very complex well and reservoir geometries, and thus the evolution of the saturation and pressure fields during a test can be reliably described. The pressure response provided by numerical simulations and the pressure derivative are then compared to the real pressure and pressure derivative trends as they evolve during injection and subsequent fall-off.

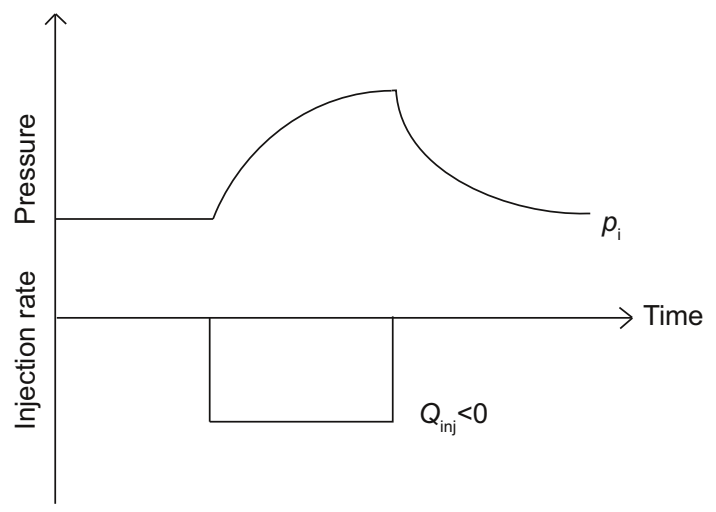

(b)

Fig. 1 Example of the draw-down/build-up sequence (a) and of the injection/fall-off sequence (b) in well testing

In the well test interpretation process, the analysis of the pressure derivative is very powerful in identifying the main features of a system because the slope of the identified linear trends or specific features such as a valley can be associated to different flow geometries (radial, linear, etc.) and thus to specific reservoir conceptual models (homogeneous or heterogeneous formation, presence of fractures or faults, etc.). Typically, analytical models are applied, each model being governed by parameters that are calibrated during the pressure and pressure derivative matching process. The parameter calibration process consists of a manual trial and error approach in which the reservoir engineer, based on experience and good judgment, changes the key parameters of the selected model until a satisfactory match is found. Alternatively, an automatic or semi-automatic numerical optimization process can be used to accelerate the matching process. The objective function can be described as the sum of the squares of the difference between real and simulated data (Welty and Miller 1979). For example, Abbaszadeh and Kamal (1988) used the least square regression algorithm to accurately match three different build-up and fall-off flow periods. In numerical well testing, a simplified reservoir model is generated and the test match is obtained by manually changing the uncertain input parameters just like in the calibration process of a conventional reservoir study. Because the information available on the reservoir is generally limited at the appraisal stage of a field when wells are typically tested, the use of optimization algorithms, e.g. evolutionary algorithms, would be unsuitable.

Provided that the selected analytical or numerical model is consistent with the reservoir under investigation, the knowledge of the model parameters provide an estimate of the well productivity and description of the formation within the test drainage area. Injection testing relies on the same pressure transient analysis as conventional tests for assessing the well productivity; therefore, it is essential that a representative reservoir model can be generated to reproduce the pressure response obtained from the real system and to subsequently calculate the pressure derivative needed for diagnostic purposes. This aspect must be kept in due consideration when setting up a model for well testing rather than for reservoir simulation. Furthermore, the analysis of the well test pressure data can often result in a non-unique solution (Kelly 1996), i.e. different models and/or sets of parameters can generate very similar pressure response. Accordingly, the uncertainty of the calibrated parameters strongly depends on the selected model and on the simplifying assumptions associated to it.

\section{Mathematical model}

In the following the standard treatment of the mathematical model is recalled (Aziz and Settari, 2002; Chen et al, 2006) so as to provide a basis for the subsequent development of the numerical model.

As previously discussed, brine, diesel or nitrogen can be pumped into oil reservoirs during injection testing (Tripaldi et al, 2009). Oil is immiscible with water but is considered to be 
miscible with diesel; however, field experience indicates that, generally, miscibility conditions do not develop within the test time (hours). This could probably be due to the limited mixing between the two fluids.

If immiscible flow conditions apply, there is no mass transfer between the injected and the reservoir fluids. Therefore, each phase needs to verify the mass conservation equation

$$
\frac{\partial\left(\phi \rho_{\alpha} S_{\alpha}\right)}{\partial t}+\nabla \cdot\left(\rho_{\alpha} \vec{v}_{\alpha}\right)+\tilde{q}_{\alpha}=0 \quad \alpha=0, \quad \mathrm{w}
$$

where $\phi$ represents the rock porosity; $\rho_{\alpha}$ is the density of each phase at reservoir conditions; $S_{\alpha}$ is the saturation of each phase; $\vec{v}_{\alpha}$ is the phase velocity and $\tilde{q}_{\alpha}$ represents the phase mass rate; $t$ is time; and the subscript $\alpha$ indicates the oil or the injection fluid.

As turbulence effects are typically negligible, in the case of liquid flow in porous media, the velocity $\vec{v}_{\alpha}$ can be expressed by extended Darcy's law, that is

$$
\vec{v}_{\alpha}=-\frac{k_{\text {eff }, \alpha}}{\mu_{\alpha}}\left(\nabla p_{\alpha}-\rho_{\alpha} g \nabla z\right)
$$

where $k_{\text {eff, } \alpha}$ represents the effective permeability to phase $\alpha ; \mu_{\alpha}$ is the phase viscosity; $g$ the gravitational constant, $\mathrm{m}^{2} / \mathrm{s} ; p$ is the pressure, and $z$ is the absolute vertical coordinate.

Substitution of Eq. (2) into Eq. (1) yields

$$
\frac{\partial\left(\phi \rho_{\alpha} S_{\alpha}\right)}{\partial t}=\nabla \cdot\left[\rho_{\alpha} \frac{k_{\mathrm{eff}, \alpha}}{\mu_{\alpha}}\left(\nabla p_{\alpha}-\rho_{\alpha} g \nabla z\right)\right]-\tilde{q}_{\alpha}
$$

In order to express Eq. (3) at stock tank conditions ( $p=1$ bar and $T=15.5^{\circ} \mathrm{C}$ ), Eq. (3) is divided by $\rho_{\alpha, \text { ST }}$

$$
\frac{\partial}{\partial t}\left(\frac{\phi S_{\alpha}}{B_{\alpha}}\right)=\nabla \cdot\left[\frac{k_{\mathrm{eff}, \alpha}}{\mu_{\alpha}}\left(\nabla p_{\alpha}-\rho_{\alpha} g \nabla z\right)\right]-q_{\alpha}
$$

where $B_{\alpha}$ is the formation volume factor, defined by the following equation

$$
B_{\alpha}=\frac{V_{\alpha, \mathrm{R}}}{V_{\alpha, \mathrm{ST}}}=\frac{\rho_{\alpha, \mathrm{ST}}}{\rho_{\alpha, \mathrm{R}}}
$$

where $V_{\alpha}$ is the volume of each fluid $\alpha$; subscripts R and ST indicate reservoir and stock tank conditions, respectively; and $k_{\text {eff }, \alpha}$ is defined as

$$
k_{\mathrm{eff}, \alpha}=k_{\mathrm{abs}} k_{\mathrm{r}, \alpha}
$$

The unknowns of the models are represented by the pressure and saturation of each phase. In order to have a wellposed problem, two other equations are needed.

Since the porous medium is fully saturated with fluids, the following equation holds

$$
\sum_{\alpha=1}^{N} S_{\alpha}=1
$$

where $N$ is the number of the phases. Eventually, the capillary pressure relation can be introduced

$$
p_{\mathrm{c}}=p_{\mathrm{o}}-p_{\mathrm{w}}
$$

Then, the boundary conditions need to be imposed. A noflow condition at the boundaries of the reservoir is assigned as a homogeneous Neumann condition

$$
\frac{\partial p}{\partial n}=0
$$

Furthermore, another Neumann conditions is imposed at the well in order to apply a fixed injection rate (injection rates are always defined as negative)

$$
-\frac{k_{\mathrm{abs}} k_{\mathrm{rw}}}{\mu_{\mathrm{w}} B_{\mathrm{w}}}\left(\nabla p_{\mathrm{w}}-\rho_{\mathrm{w}} g \nabla z\right) \cdot \hat{n}=Q
$$

Finally, making Eq. (4) explicit for both phases and introducing Eqs. (8) and (7) into Eq. (4), it is possible to formulate a mathematical model able to describe the physical phenomena occurring during an injection test in an oil reservoir as

$$
\frac{\partial}{\partial t}\left(\frac{\phi S_{\mathrm{w}}}{B_{\mathrm{w}}}\right)=\nabla \cdot\left[\frac{k_{\mathrm{abs}} k_{\mathrm{rw}}}{\mu_{\mathrm{w}} B_{\mathrm{w}}}\left(\nabla p_{\mathrm{w}}-\rho_{\mathrm{w}} g \nabla z\right)\right]-q_{\mathrm{w}}
$$

$\frac{\partial}{\partial t}\left(\frac{\phi\left(1-S_{\mathrm{w}}\right)}{B_{\mathrm{o}}}\right)=\nabla \cdot\left[\frac{k_{\mathrm{abs}} k_{\mathrm{ro}}}{\mu_{\mathrm{o}} B_{\mathrm{o}}}\left(\nabla p_{\mathrm{w}}+\nabla p_{\mathrm{c}}-\rho_{\mathrm{o}} g \nabla z\right)\right]-q_{\mathrm{o}}$

with the boundary conditions expressed by Eqs. (9) and (10).

\section{Numerical model}

A mathematical model describing two-phase flow in a porous medium is introduced in the previous section. In this section a discretization of the model using the FVM is proposed by the authors following the method suggested by Leveque (2002) for the hyperbolic equations. The developed numerical model provides a solution of the PDE's and also applies to very complex geometries.

Let $V_{i}$ be a control volume. Integrating Eq. (1) over $V_{i}$ yields

$$
\begin{aligned}
& \frac{\partial}{\partial t} \int_{V_{i}} \phi \rho_{\alpha} S_{\alpha} \mathrm{d} V+\int_{V_{i}} \nabla \cdot\left(\rho_{\alpha} \vec{v}_{\alpha}\right) \mathrm{d} V+\int_{V_{i}} \tilde{q}_{\alpha} \mathrm{d} V=0 \\
& \alpha=\mathrm{o}, \quad \mathrm{w}
\end{aligned}
$$

Using the divergence theorem, it is possible to express the volume integral as a surface integral, then

$$
\frac{\partial}{\partial t} \int_{V_{i}} \phi \rho_{\alpha} S_{\alpha} \mathrm{d} V+\int_{V_{i}} \nabla \cdot\left(\rho_{\alpha} \vec{v}_{\alpha}\right) \cdot \hat{n} \mathrm{~d} S+\int_{V_{i}} \tilde{q}_{\alpha} \mathrm{d} V=0
$$

where $\partial V_{i}$ is the frontier or interface of the control volume.

Dividing Eq. (14) by $\rho_{\alpha, \text { sT }}$

$$
\frac{\partial}{\partial t} \int_{V_{i}} \frac{\rho_{\alpha}}{\rho_{\alpha, \mathrm{ST}}} \phi S_{\alpha} \mathrm{d} V+\int_{V_{i}} \frac{\rho_{\alpha}}{\rho_{\alpha, \mathrm{ST}}} \vec{v}_{\alpha} \cdot \hat{n} \mathrm{~d} S+\int_{V_{i}} \frac{\tilde{q}_{\alpha}}{\rho_{\alpha, \mathrm{ST}}} \mathrm{d} V=0
$$


with

$$
\begin{aligned}
& \frac{\rho_{\alpha}}{\rho_{\alpha, \mathrm{ST}}}=\frac{m_{\alpha}}{V_{\mathrm{R}}} \frac{V_{\mathrm{ST}}}{m_{\alpha}}=\frac{1}{B_{\alpha}} \\
& \frac{\partial}{\partial t} \int_{V} \frac{\phi S_{\alpha}}{B_{\alpha}} \mathrm{d} V+\int_{\partial V} \frac{1}{B_{\alpha}} \vec{v}_{\alpha} \cdot \hat{n} \mathrm{~d} S+\int_{V} q_{\alpha} \mathrm{d} V=0 \\
& \frac{\tilde{q}_{\alpha}}{\rho_{\alpha, \mathrm{ST}}}=\frac{m_{\alpha}}{V_{\mathrm{R}} \Delta t} \frac{V_{\mathrm{ST}}}{m_{\alpha}}=\frac{1}{B_{\alpha} \Delta t}=q_{\alpha}
\end{aligned}
$$

Considering a single cell $i$,

$$
\frac{\phi_{i} S_{\alpha_{i}}}{B_{\alpha_{i}}}=\frac{1}{V_{\mathrm{c}}} \int_{V_{i}} \frac{\phi S_{\alpha}}{B_{\alpha}} \mathrm{d} V
$$

where $V_{\mathrm{c}}$ is the cell volume

$$
F_{k}=\frac{1}{A_{k}} \int_{A_{k}} \frac{1}{B_{\alpha}} \vec{v} \cdot \hat{n}_{k} \mathrm{~d} S_{k} \rightarrow \int_{\partial V} \frac{1}{B_{\alpha}} \vec{v} \cdot \hat{n}_{k} \mathrm{~d} S=\sum_{k} A_{k} F_{k}
$$

where the interface is expressed as a set of discrete interfaces as

$$
\partial V=U_{k} A_{k}
$$

The rate in a single cell is expressed as

$$
Q_{\alpha_{i}}=\int_{V_{i}} q_{\alpha} \mathrm{d} V
$$

and the final balance equation for a single cell is stated as follows

$$
V_{\mathrm{c}} \frac{\mathrm{d}}{\mathrm{d} t}\left(\frac{\phi_{\alpha} S_{\alpha_{i}}}{B_{\alpha_{i}}}\right)+\sum_{k} A_{k} F_{k}+Q_{\alpha_{i}}=0
$$

This set of equations is developed for each phase and for each single cell in the model. This leads to a set of nonlinear algebraic equations, which are usually solved using a Newton-like method, in which the stencil of the Jacobian matrix depends on the grid.

\section{Case studies}

Four synthetic cases are presented and discussed to prove the effectiveness and flexibility of the FVM to simulate conventional tests as well as injection tests. In the first case, a conventional production-build up test in a horizontal well was simulated using an analytical model, the FDM in a structured Cartesian grid and the FVM in a radial grid. In the second case, an injection test in a horizontal well was simulated using the FDM and the FVM; analytical models could not be used because, as already discussed, they are not able to properly describe injection tests, especially in the case of complex geometries. In the third case study, an injection test in the presence of permeability barriers was simulated; finally, in the last case an injection test in a heterogeneous formation was simulated. The last two case studies were simulated only using the FVM.
Commercial software (Eclipse, Schlumberger) was used to carry out the simulations with the FDM; the software used to perform the simulations with the FVM was developed in-house. The analytical pressure transient response was generated with a commercial tool for well testing (Saphir, Kappa).

A number of simplifying hypotheses had to be adopted in order to achieve a meaningful comparison among the different models. For this reason, capillary pressures and thermal effects were neglected.

The simulation results were compared so as to highlight the significant differences among the model responses.

\subsection{Synthetic reservoir model}

In the first model, a homogeneous and isotropic oilbearing formation was tested through a horizontal well. The physical domain consisted in a rectangular reservoir with a length of $2,000 \mathrm{~m}$, a width of $1,000 \mathrm{~m}$ and a thickness of $40 \mathrm{~m}$. The porosity was assumed equal to 0.2 , the irreducible water saturation equal to 0.2 and the absolute permeability equal to $100 \mathrm{mD}$. The maximum relative permeability to oil was 0.8 while the maximum relative permeability to water was 0.4 . The well was placed in the middle of the pay thickness and in the center of the physical domain. The perforated interval of the well was set equal to $200 \mathrm{~m}$. The initial pressure of the reservoir at the datum depth of 3,000 $\mathrm{m}$ was assumed to be 300 bar. The wellbore radius was $0.1 \mathrm{~m}$ and the rock compressibility was set equal to $4.5 \times 10^{-5} \mathrm{bar}^{-1}$.

For the sake of simplicity, zero mechanical skin was imposed. No-flow boundaries were assumed at the limits of the physical domain. Oil and water were modeled as slightly compressible fluids. The adopted compressibilities were $4.7 \times 10^{-4}$ bar $^{-1}$ and $4.0 \times 10^{-5}$ bar $^{-1}$ for oil and water, respectively. The formation volume factor at the initial pressure was assumed equal to $1.3 \mathrm{~m}^{3} / \mathrm{m}^{3}$ and to $1.0 \mathrm{~m}^{3} / \mathrm{m}^{3}$ for oil and water (at standard temperature), respectively. Fluid viscosities were assumed to be constant and equal to $0.2 \mathrm{cP}$ and $0.4 \mathrm{cP}$ for oil and water, respectively.

\subsection{Finite difference discretization}

The numerical grid consisted in a 3D Cartesian mesh with hexahedral cells, whose faces are parallel to the Cartesian axis. The number of cells was 50 in the $x$ direction, 100 in the $y$ direction and 50 in the $z$ direction. The horizontal well was positioned along the $y$ direction.

In order to assess the impact of the volume discretization in the FDM, two different grids were generated: the first one was obtained imposing a regular discretization of the reservoir volume (Fig. 2(a)) with $20 \mathrm{~m} \times 20 \mathrm{~m}$ cells in the horizontal plane. In the second grid, a refinement in the near wellbore zone was applied (Fig. 2(b)). A geometric progression of the cell dimensions was used from the well cells in the $x$ and $z$ directions: the cell dimensions varied from $3 \mathrm{~cm}$ to $150 \mathrm{~m}$. In the $y$ direction, a uniform discretization was applied along the well, but a geometric progression of the cell dimensions was adopted starting from the heel and toe of the well. This kind of grid allows achievement of a more accurate response with higher resolution at the beginning of the test, when the 
pressure disturbance has propagated for a limited distance from the well in the reservoir.

In the FDM, the well is a source/sink term in the continuity equation. Therefore, the well response is simulated through a well model; the most popular well model is the Peaceman equation (Peaceman, 1978).

The time domain was also discretized using a geometric progression: an initial timestep of 1 second was set in order to guarantee the convergence of the non-linear solver based on the smallest cell volume; then, a progression ratio was adopted.

The selected discretization was restarted at the beginning of each flow period. This timestep progression ensured a good compromise between resolution and computational time; while at the same time, it facilitated the convergence of the numerical system, especially at early time of each flow period when changes in pressure and saturation are substantial. It also avoided numerical oscillations of the pressure derivatives, thus, simulation results could be examined according to the classical well test interpretation approach.

\subsection{Finite volume discretization}

The reservoir was also modeled using a near-wellbore, numerical simulator, developed by the authors, based on the FVM. The numerical grid consisted of a cylindrical 3D mesh, where the cell dimensions increased geometrically in the radial direction (Fig. 2(c)). In the direction of the well axis, the reservoir volume was divided in a similar way to the finite difference model.
The well pressure was approximated with the pressure of the first cell in the radial direction. This assumption is valid whenever the first cell is very small because the pressure drop between the center of the cell and the sand-face can be neglected.

The flexibility of the FVM allows simulation of the well, both with small cells corresponding to the real well dimensions or with the aid of a well model in place of an inner boundary condition. However, the well model is not needed because it does not provide an appreciable accuracy improvement, thus, the computational complexity can be reduced.

Since no well model was used, the rates were imposed as fluxes at the internal faces of the innermost cells. This is equivalent to having a source term in the cells that are open to flow, i.e. through the perforated interval.

The same time discretization was adopted as in the FDM. An initial timestep of 1 second was set in order to guarantee the convergence of the non-linear solver; then, the same progression ratio as in the spatial domain was used. In order to solve the system of non-linear algebraic equations arising from the FVM discretization, an inexact Newton method was used (Klie et al, 1996; Nash and Sofer, 1996). The Newton method requires solving a linear system of algebraic equations at each iteration. Based on the grid, the stencil of the Jacobian matrix in the case of two-phase flow, i.e. injection test, is a block heptadiagonal non-symmetric matrix. The matrix is very large, sparse and ill conditioned.

In the case of single phase flow, the conditioning of the

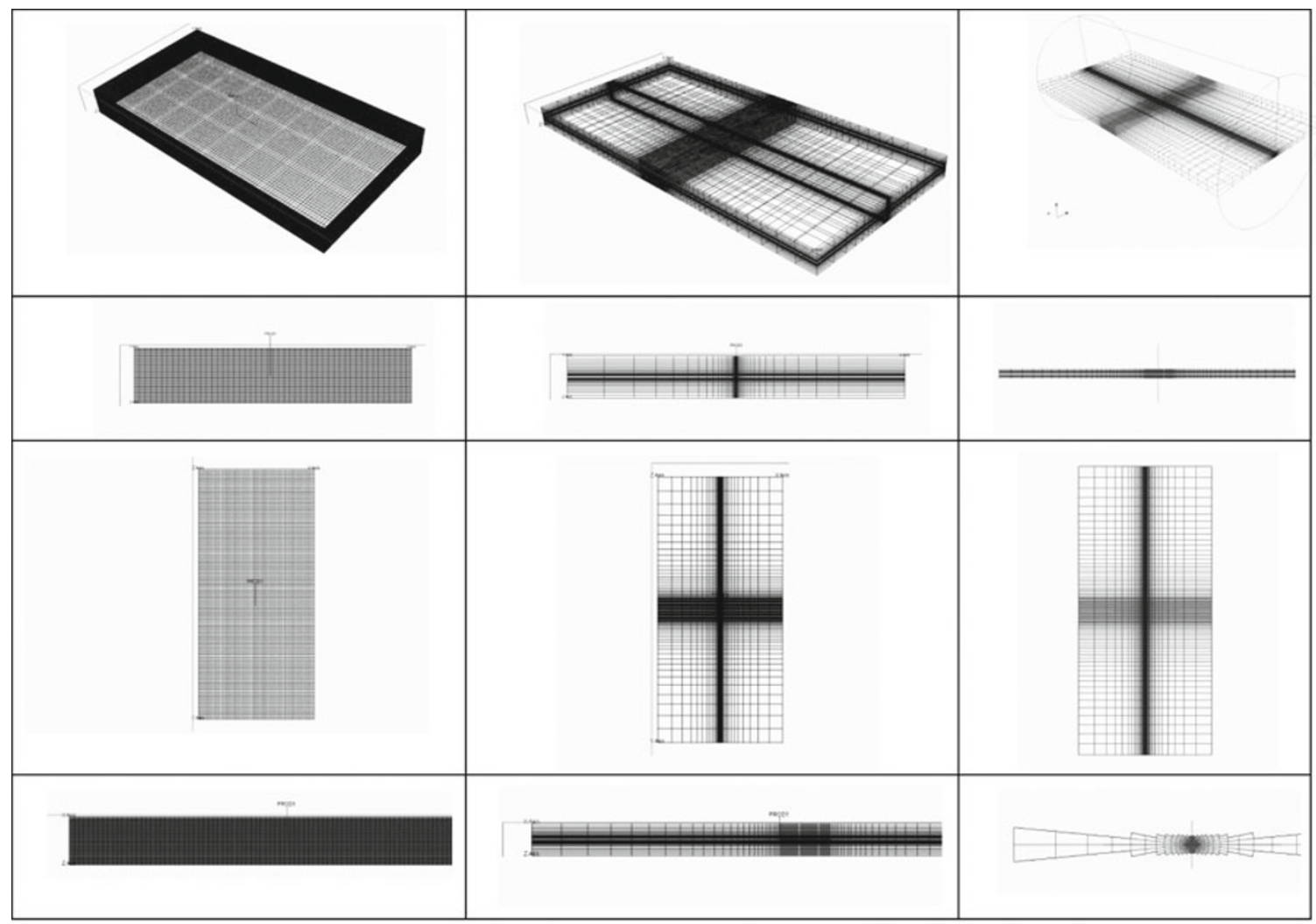

(a)

(b)

(c)

Fig. 2 Coarse finite difference grid (a), refined finite difference grid (b) and finite volume grid (c) 
matrix is substantially lower. The matrix stencil is a simple heptadiagonal matrix and the number of unknowns is reduced by a half because the saturations do not need to be calculated.

When the number of cells is large, direct methods, i.e. the Gauss method, cannot be applied and iterative methods are used. A very efficient iterative solver based on Krylov-spaces known as GMRES was adopted in the model discussed in this paper. Further information about GMRES can be found in the work developed by Saad and his coworker (Saad and Schultz, 1986; Saad, 1993) and Van der Vorst and Vuik (1994).

In the model, the preconditioner used for the GMRES was different for the production and the injection test, respectively. A classical preconditioner based on the incomplete lower-upper factorization (ILU) (Behie, 1985; Xing and Ma, 1996) was used to simulate the production test, whereas the preconditioner was changed according to three phenomenologically different periods occurring during an injection test (Fig. 3).

During the early injection phase, where substantial changes in pressure and saturation occur, the two-stage preconditioner proposed by Klie et al (1996) was applied. At late time, where the saturation front is still moving but the pressure changes are moderate, it is sufficient to use a simple ILU preconditioner. In the fall-off period, where the water front is almost stationary, again it is possible to efficiently precondition the Jacobian matrix with an ILU preconditioner.

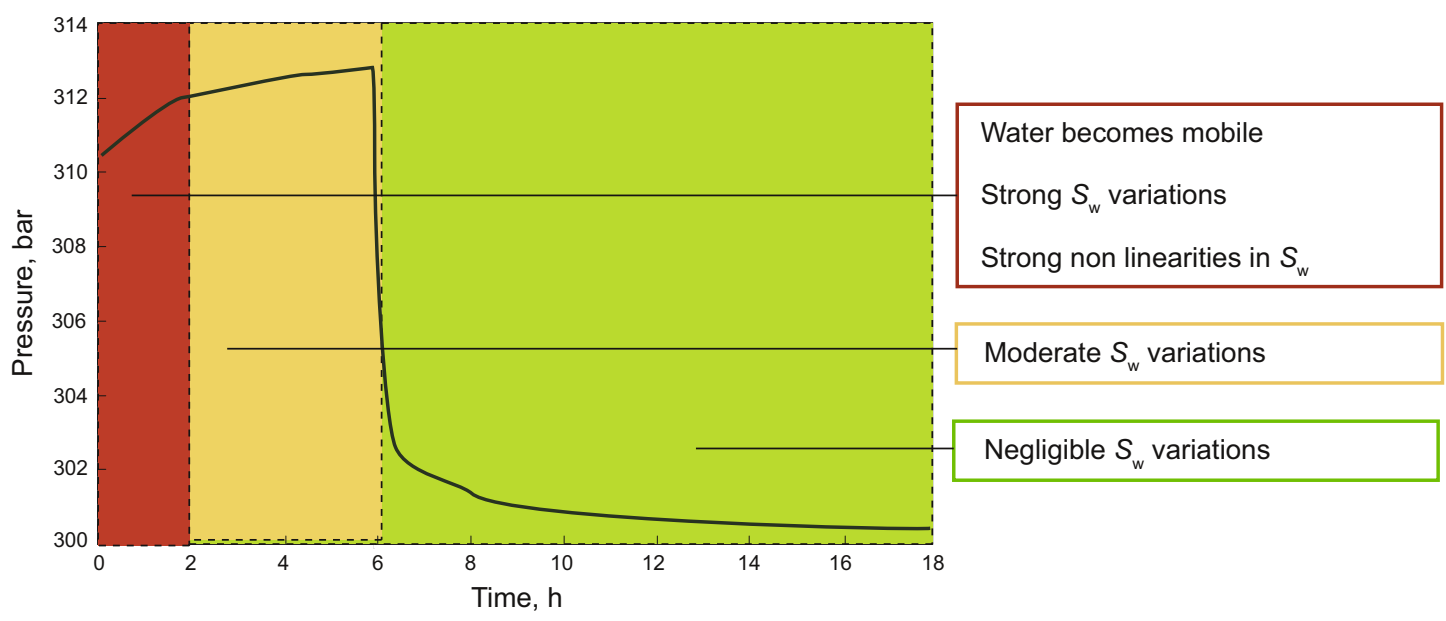

Fig. 3 Identification of the three phenomenologically different periods during an injection test

\subsection{Analytical model}

In the case of horizontal wells, the pressure variation in the time following the start of production, propagates radially in the vertical plane orthogonal to the well trajectory. The behavior of the well can be compared to that of a vertical well producing from a reservoir with a thickness equal to the length of the horizontal segment open to flow. Therefore, the pressure derivative exhibits a horizontal stabilization, which is a function of the formation vertical and horizontal permeability and of the fluid viscosity. Once the pressure disturbance has reached the upper and lower boundaries of the level, a linear flow - perpendicular to the well axis- develops along the producing layer provided that the horizontal segment of the well is greater than the thickness of the producing layer. If the duration of the test is long enough, it is eventually possible to recognize a second radial flow, called pseudoradial, which develops in the horizontal plane (Bourdet, 2002). The stabilization of the pressure derivative depends on the formation horizontal permeability and on the fluid viscosity. In an ideal situation, in which all the flow geometries that can establish during the test are recognizable, the trend of the pressure derivative on the diagnostic plot would be described by a first horizontal stabilization, then by a line with a $1 / 2$ slope, and finally by a second horizontal stabilization (Fig. 4).

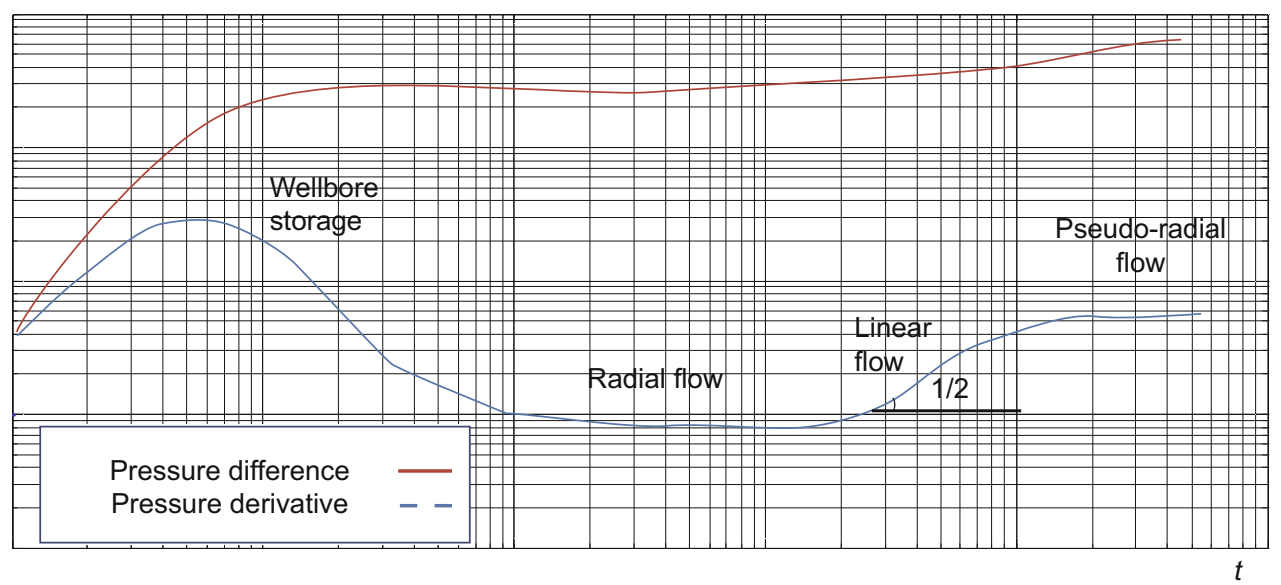

Fig. 4 Pressure change and derivative for a horizontal well in an ideal case 
The duration of each flow geometry depends on the length of the horizontal well with respect to the thickness of the producing layer, on the position of the well in the layer and on the petrophysical characteristics of the reservoir. Furthermore, it should be pointed out that the well response can be different from the expected trend. This is because the flow geometry may not last long enough to be clearly detectable or it can be partially masked by wellbore storage effects, by the flow effects at the ends of the well, and by the heterogeneity of the reservoir (Joshi, 1991; Kuchuk, 1995).

The analytical model used for comparison is the uniform flux horizontal well model. The literature is very rich with discussion on the preferred approach to simulate a finite horizontal well (Babu and Odeh, 1989; 1992; Peaceman, 1990; Brigham, 1990; Suprunowicz and Butler, 1992). It is generally agreed that the constant pressure condition is more close to the physical reality, but the uniform flux boundary has the advantage that it can be easily incorporated into software for fluid flow simulation (Zhan, 1999). In the uniform flux model, the inflow of fluids from the reservoir into the well is assumed to be uniform over the length of the well, so the pressure varies along the same well length (Ozkan and Raghavan, 1989; 1991; Joshi, 1991).

With $L_{\mathrm{w}}$ being the length of the well open to production, the interpretation of a conventional production test in a horizontal well yields:

$k_{z}$ vertical permeability, based on the first horizontal stabilization of the pressure derivative (radial flow in the vertical plane) yielding $L_{\mathrm{w}} \sqrt{k_{x y} k_{z}}$.

$k_{x y}$ horizontal permeability, based on the second horizontal stabilization of the pressure derivative (pseudo-radial flow in the horizontal plane) yielding $k_{x y} h$, where $h$ is the reservoir net pay.

$S_{t}$ total skin, which is a combination of the well skin $S_{\mathrm{TV}}$ and an additional geometrical skin $S_{\mathrm{c}}$ (Bourdet, 2002):

$$
S_{\mathrm{t}}=S_{\mathrm{TV}} \frac{h}{L_{\mathrm{w}}} \sqrt{\frac{k_{x y}}{k_{z}}}+S_{\mathrm{c}}
$$

The well skin is due to permeability damage $\left(S_{\mathrm{w}}\right)$ referred to the producing well length, minus a correction to account for anisotropy:

$$
S_{\mathrm{TV}}=S_{\mathrm{w}}-\ln \left[0.5\left(\sqrt[4]{\frac{k_{x y}}{k_{z}}}+\sqrt[4]{\frac{k_{z}}{k_{x y}}}\right)\right]
$$

The negative geometrical skin $S_{\mathrm{c}}$ accounts for partial penetration of the well in the producing formation and is given by (Kuchuk et al, 1991; Bourdet, 2002):

$$
\begin{aligned}
S_{\mathrm{c}}= & \ln \left(\frac{4 r_{\mathrm{w}}}{L_{\mathrm{w}}}\right)-1.151 \sqrt{\frac{k_{x y}}{k_{z}}} \frac{4 h}{L_{\mathrm{w}}} \log _{10}\left[\frac{\pi r_{\mathrm{w}}}{h}\left(1+\sqrt{\frac{k_{z}}{k_{x y}}}\right) \sin \left(\frac{\pi r_{\mathrm{w}}}{h}\right)\right] \\
& -\frac{1}{2} \frac{k_{x y}}{k_{z}} \frac{4 h^{2}}{L_{\mathrm{w}}^{2}}\left(\frac{1}{3}-\frac{z_{\mathrm{w}}}{h}+\frac{z_{\mathrm{w}}^{2}}{h^{2}}\right)
\end{aligned}
$$

where $r_{\mathrm{w}}$ is the well radius.

Given that isotropic conditions were assumed in the simulated reservoir and that the horizontal well had no standoff (the well is centered in the reservoir pay), the well skin reduces to $S_{\mathrm{w}}$ and the geometrical skin can be expressed as:

$$
S_{\mathrm{c}}=\ln \left(\frac{4 r_{\mathrm{w}}}{L_{\mathrm{w}}}\right)-\frac{h}{L_{\mathrm{w}}} \ln \left(\frac{2 \pi r_{\mathrm{w}}}{h}\right)
$$

Therefore, the "true" mechanical skin of the well can be calculated from the total skin obtained from the test interpretation.

\subsection{Case 1-Production test with single phase flow}

In order to test the performance of the FDM and of the FVM previously introduced, a classical production test where single phase flow occurs was simulated. The test consisted of two flow periods: a rate of $1,000 \mathrm{~m}^{3} /$ day of oil was initially produced for 24 hours; then the well was shut-in for 24 hours. The pressure response at the well was simulated assuming that the gauge was positioned at the depth of the horizontal drain of the well.

The reservoir pressure response during the test is shown in Fig. 5 for the analytical model, FDM (coarse and fine grids) and FVM. The pressure change and derivative on the log-log diagnostic plot for the build-up period show the typical trend for a horizontal well (Fig. 6). In terms of pressure derivative, the FVM correlates very well to the analytical model, whereas the FDM with the fine grid, although showing a good match, appears to be less accurate than the FVM, particularly during the first timesteps. In the case of the FDM with the coarse grid, the first horizontal stabilization of the pressure derivative, indicating radial flow in the vertical plane, cannot be identified. The anomalous pressure derivative trend is due to the lack of resolution of the model grid. Results also show that the cylindrical grid used for the FVM better reproduces the radial flow governing the early production phase because at least two block faces are orthogonal to the main flow direction and; therefore, it was also confirmed that the use of a well model could be avoided.

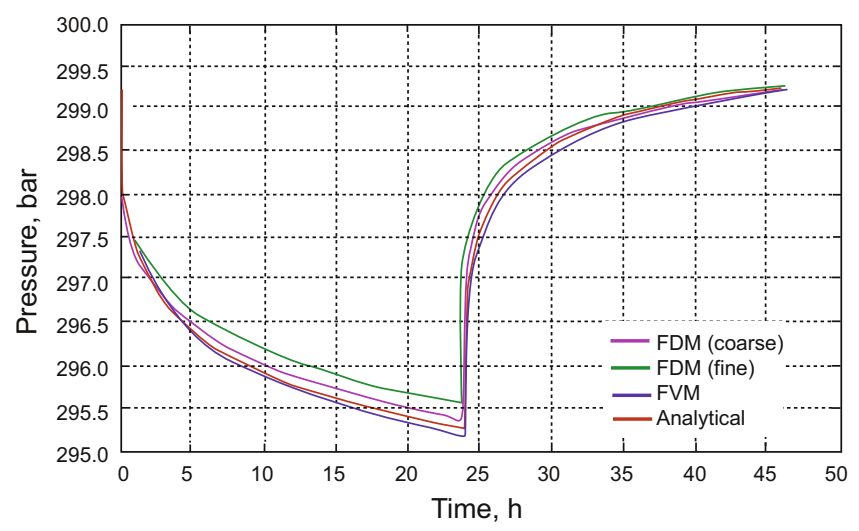

Fig. 5 Case 1-Comparison of the pressure history simulated with different models during the production test 


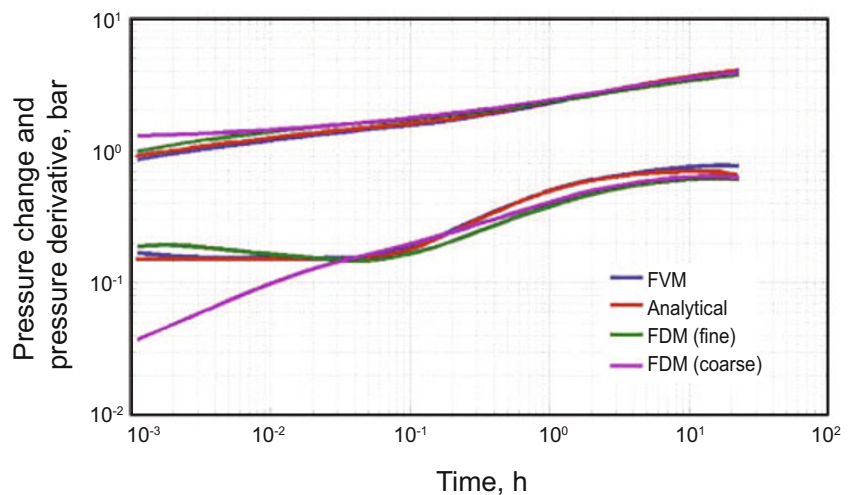

Fig. 6 Case 1-Pressure change and derivative simulated with different models during the build-up period

\subsection{Case 2-Injection test}

The second test consisted of an unconventional well test in which $1,000 \mathrm{~m}^{3} /$ day of water were injected into the reservoir for 24 hours. The well was then shut-in for a 24-hours falloff period. The injected water was assumed to have the same properties as the reservoir water. The pressure response at the well was simulated assuming that the gauge was positioned at the depth of the horizontal drain of the well.

Since no analytical model is able to describe the physical phenomena occurring in the reservoir during injection, only the numerical models could be used to simulate the test. However, thermal gradients induced by injection of cold water in the reservoir were neglected because the commonly used reservoir simulators assume the system to be isothermal and do not provide the thermal option. Furthermore, inclusion of thermal effects would not have been relevant to the authors, whose interests are focused on demonstrating the appropriateness of the FVM to describe two-phase flow in the case of a horizontal well.

As it can be observed from the comparison of the pressure history obtained with the different models (Fig. 7), the FDM with the fine grid and the FVM provide a very similar response. On the other hand, the FDM with the coarse grid completely fails to reproduce the actual behavior of the physical system and in particular, the pressure rises during the injection phase. The main reason for this difference is that the well model implemented in the typical reservoir simulator is unable to handle the phenomena occurring in the near wellbore zone. When using the fine grid FDM, the lack of accuracy evident for the coarse grid FDM is not present because the fine grid around the well allows proper description of the two-phase flow, given the adopted time discretization. Additionally, the difference between the well cell pressure and the bottom hole pressure is proportional to the dimension of the grid cell where the well is located. The FVM does not require the use of a well model because all the physical phenomena are simulated numerically and an accurate pressure response can be obtained. The comparison of the pressure derivative for the FVM and fine grid FDM during the fall-off period is shown in Fig. 8.

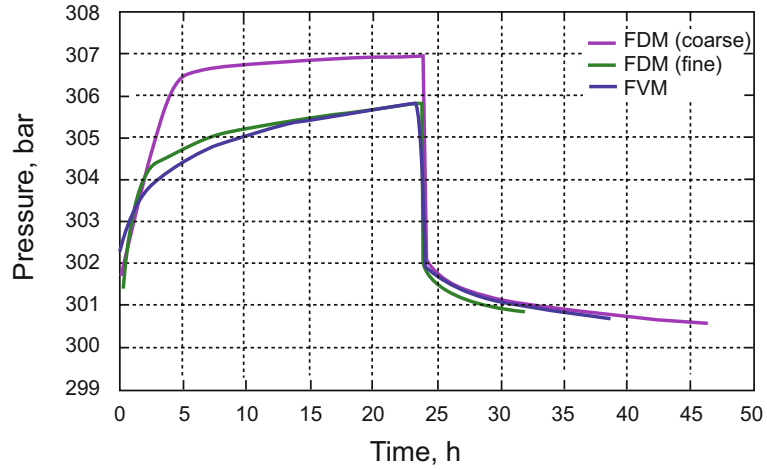

Fig. 7 Case 2-Comparison of the pressure history during the injection test

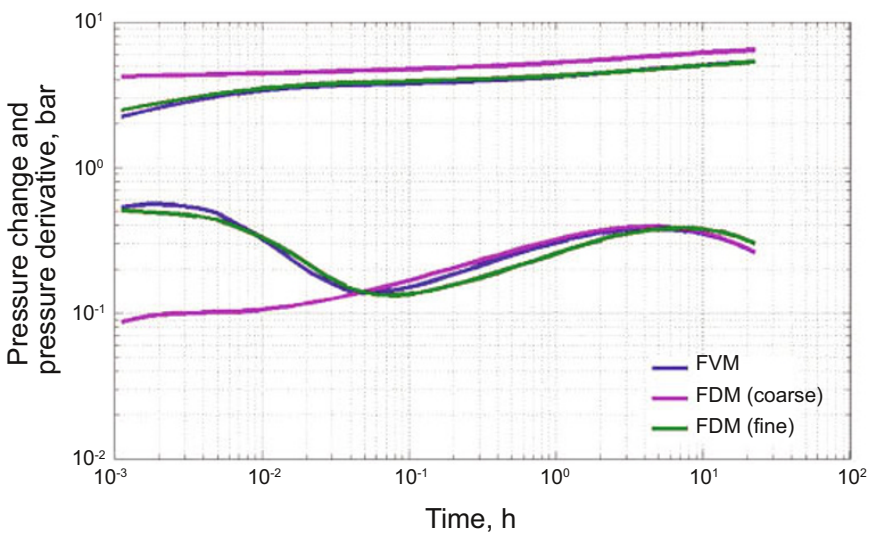

Fig. 8 Case 2-Pressure change and derivative simulated with different models during the fall-off period

The derivative of the pressure response simulated with the FVM clearly shows a first horizontal stabilization, which is only approximately reproduced by the fine grid FDM, during the early time of the fall-off period (Fig. 8). This stabilization corresponds to radial flow in the vertical plane (see also Fig. 9) and is characterized by the mobility of the injected fluid (water), which has displaced the hydrocarbon originally in place in the near wellbore zone. Then a transition occurs and a second horizontal stabilization can be observed, which once again corresponds to radial flow; however, this time it is characterized by the mobility of the mobile reservoir fluid (oil). Afterwards, linear flow develops in the reservoir, as very well captured by the FVM (see also Fig. 10), and the slope of the pressure derivative approaches $1 / 2$. Finally, pseudo-radial flow in the horizontal plane develops (see also Fig. 11). It should also be noted that the all boundaries of the reservoir are detected within the test time. This was not the case when the well was produced, although the test time was the same. The different time at which the boundaries are "seen" on the pressure derivative plot depends on the different properties of the system (namely, fluid viscosity and total compressibility) in the case of the production test (where the only fluid was oil) and in the case of the injection test (when water has flooded a portion of the reservoir). This is no surprise because the effect of fluid viscosity and total system compressibility on the time needed to detect possible reservoir boundaries is 


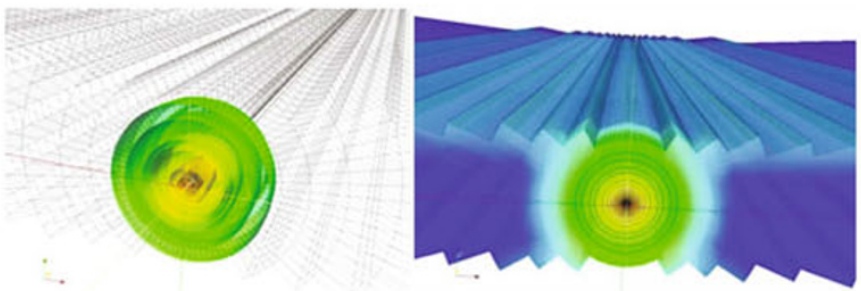

Fig. 9 Case 2-Radial flow in the vertical plane at the early time

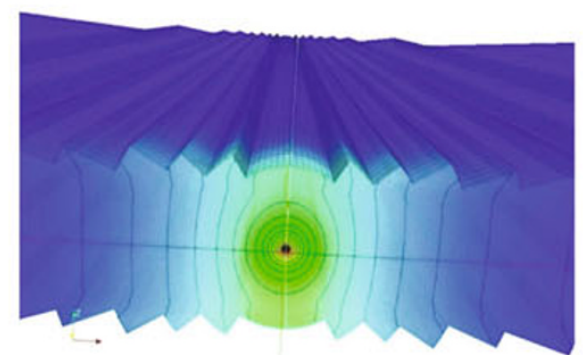

Fig. 10 Case 2-Linear flow at the middle time

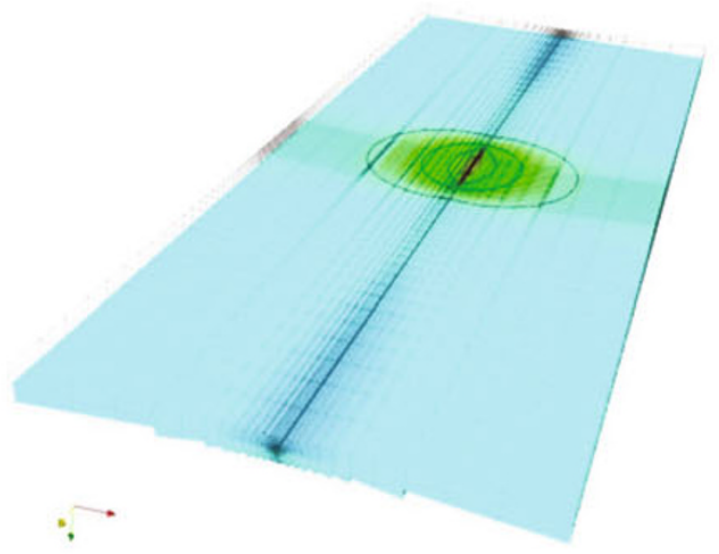

Fig. 11 Case 2-Pseudo-radial flow on the horizontal plane

well known from the well testing theory (Bourdet, 2002).

The possibility to correctly identify the first horizontal stabilization is essential to evaluate the mechanical skin, which affects the well productivity. In fact, the skin associated to the second stabilization, which is much larger that the mechanical skin, also comprises a component which is due to the presence of two mobile fluids in the reservoir (water and oil) and which is bound to disappear when the well is produced. The skin effect is also quite evident by comparing the pressure change and derivative in Figs. 6 and 8 .

The pressure derivative simulated with the coarse grid FDM is acceptable at middle and late time only, because the trend at early time cannot reproduce the saturation changes in the reservoir as a consequence of injection. Therefore, it would be impossible to determine the skin induced by permeability damage and thus the well productivity.

\subsection{Case 3-Injection test with permeability barriers}

In this model, permeability barriers, i.e. two leaky faults, were placed in the physical domain. The transmissibility across the faults was $10 \mathrm{mD}$. The first fault was positioned parallel to the injection well at a distance of $170 \mathrm{~m}$ and the second orthogonal to the well at a distance of $400 \mathrm{~m}$. The other model characteristics and the rock and fluid properties were the same as in case 2 , taken as a reference. A representation of the physical model is shown in Fig. 12. Again, a rate of $1,000 \mathrm{~m}^{3} /$ day of water was initially injected into the reservoir for 24 hours. The well was then shut-in for a 24-hours fall-off period.

The pressure field at the end of the injection period, clearly showing how the pressure front has reached the permeability barriers altering the pseudo-radial flow, is shown in Fig. 13. As expected, the pressure derivative of the fall-off period is affected by the presence of transmissibility reductions close to the well (Fig. 14), as it is evident by comparison with the system response obtained in the absence of any barrier (case 2).
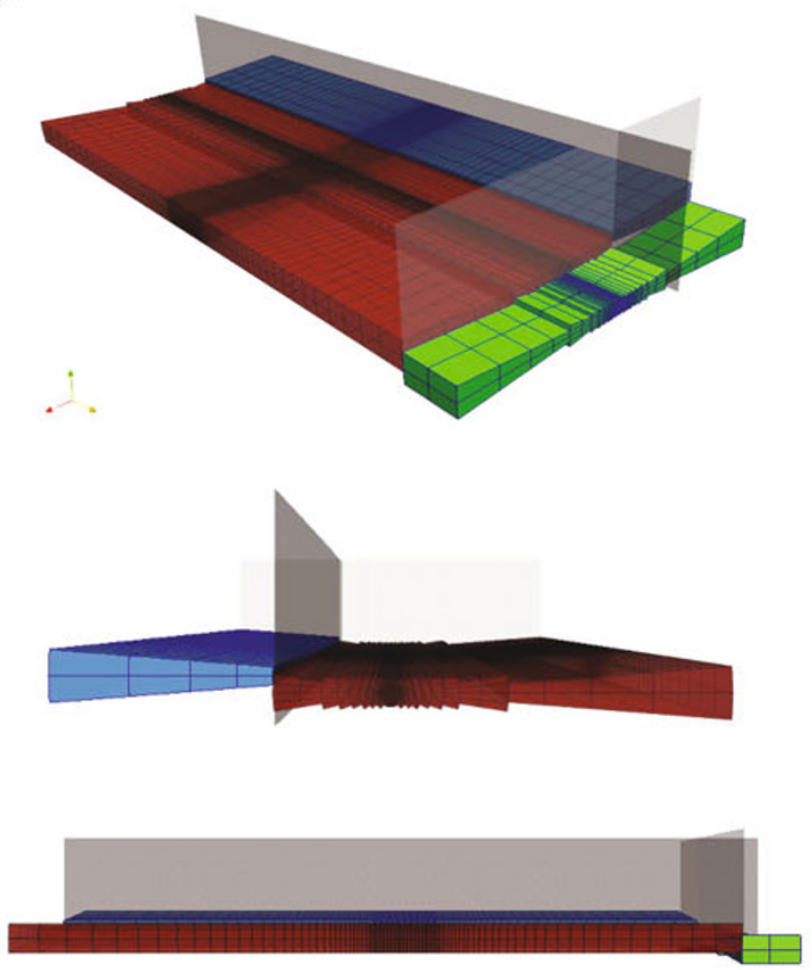

Fig. 12 Case 3-Horizontal well in a faulted formation

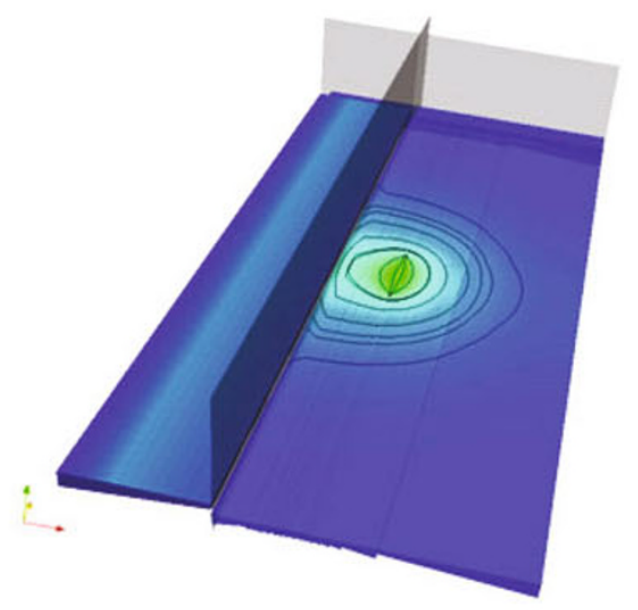

Fig. 13 Case 3-Pressure field at the end of the injection period 


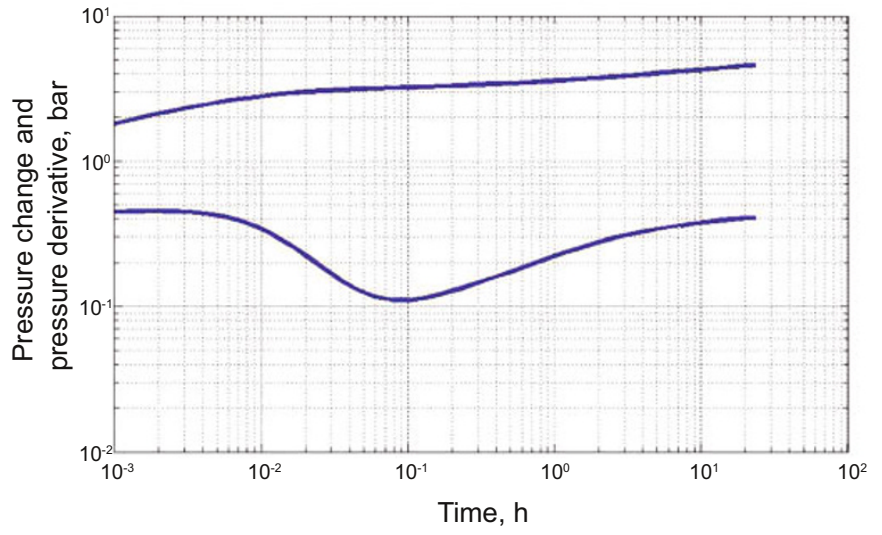

Fig. 14 Case 3-Pressure change and derivative simulated with the FVM during the fall-off period

\subsection{Case 4-Injection test with heterogeneous permeability field}

In the last case considered, the oil reservoir was comprised of three different permeability regions, ranging from 50 to $150 \mathrm{mD}$. The permeability field is shown in Fig. 15. All the other model properties were the same as in reference case 2 . Also, the same rate history was adopted.

The pressure change and derivative curves corresponding to the fall-off period are plotted in Fig. 16. Because the permeability variations are limited, the different flow regimes, namely radial flow in the water flooded zone, linear flow and, finally, pseudo-radial flow in the undisturbed oil zone can be recognized, and the horizontal stabilizations are only slightly different from those of the reference case (case 2). However, not all the reservoir boundaries can be detected during the test time due to the presence of the low permeability region.

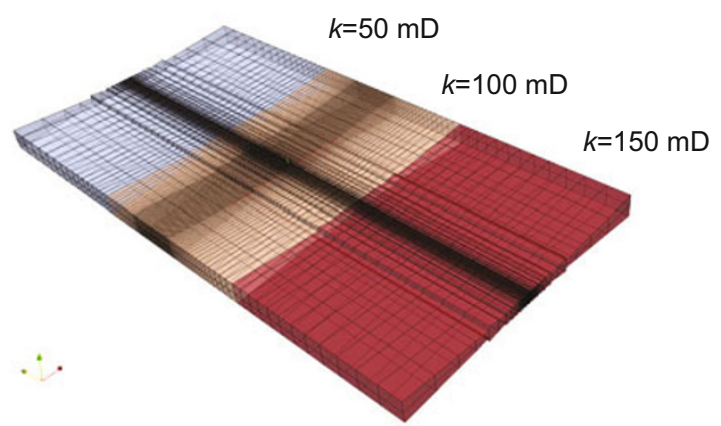

Fig. 15 Case 4-Horizontal well in a heterogeneous formation

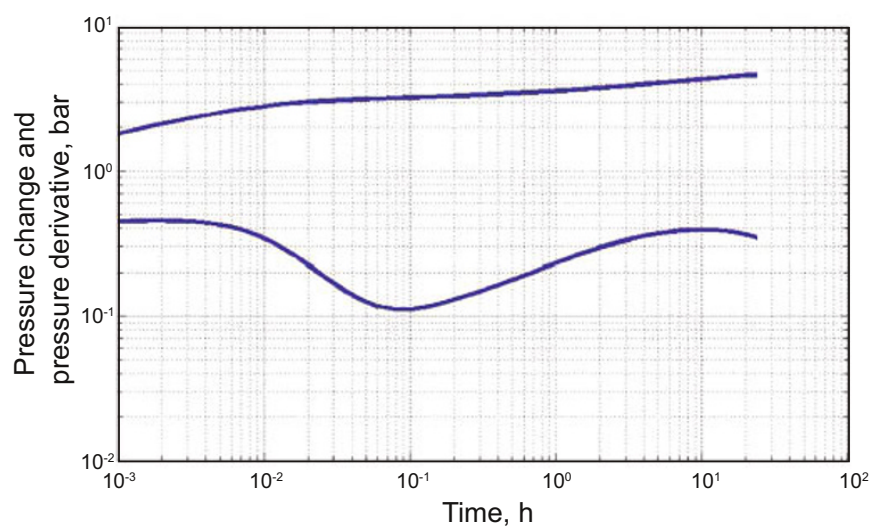

Fig. 16 Case 4-Pressure change and derivative simulated with the FVM during the fall-off period

\section{Conclusions}

In this paper, a general framework for the simulation of complex fluid flow phenomena in porous media was presented. The study was triggered by the need for a numerical model with the ability to correctly simulate injection tests in hydrocarbon reservoirs. Well testing is an essential and irreplaceable methodology for dynamic reservoir characterization and the evaluation of the potential productivity of oil and gas wells. Injection testing is one of the emerging unconventional methodologies, and is very attractive because it does not require surface production and flaring; but it poses new challenges to the ability of correctly simulating the evolution of the saturation and pressure fields in time in the presence of gravitational and thermal effects.

A finite volume model (FVM) with a radial gridding in the vertical plane was developed for describing the near-wellbore two-phase fluid flow in oil reservoirs as a result of injecting water or diesel (which also behaves as an immiscible fluid under the test conditions). The developed model was initially applied to simulate the pressure response during a conventional well test of a horizontal well draining an oil reservoir. In this simple case, the oil is produced and only mono-phase flow occurs in the reservoir. The results obtained with the FVM were compared with those provided by the uniform flux horizontal well analytical model, taken as a reference. In this way, the FVM's ability to correctly describe the physical phenomena occurring in the reservoir and to provide an accurate well pressure response was proved. The same test was also simulated with the aid of a finite difference reservoir simulator, using both a relatively coarse grid and a fine grid. Subsequently, the more complex case of injection tests were studied. Water injection in the oil reservoir through the same horizontal well was simulated with the FVM and with the finite difference model. Analytical models could not be applied as they neglect gravitational forces, thermal effects and capillary pressures, which can strongly affect the pressure response from the reservoir.

As expected, the finite difference model did not lead to representative results when a regular, relatively coarse grid (having $20 \mathrm{~m} \times 20 \mathrm{~m}$ cells in the horizontal plane) was used. A reliable response could only be obtained when using optimized local grid refinements and optimal time stepping. It might be claimed that the accuracy of the finite difference solution might have been further improved by using advanced gridding options, such as adaptive or hybrid grids which would conform to the geometry of the well around the well. However, the tuning procedures are difficult to accomplish. They would require specialized expertise to make the right grid adjustments, additional numerical work and, mostly, prior knowledge of the expected outcome. On the other hand, the FVM is much more robust because it is independent of the gridding options because the discretization is performed in the physical space.

The FVM was able to accurately reproduce the pressure behavior even in complex situations, i.e. in the presence of heterogeneities and permeability barriers, proving the flexibility of the method when simulating non-conventional tests. Unlike analytical models, providing solutions only 
for a relatively restricted set of simplified cases if compared to the possibilities offered by directional drilling and unconventional well tests, the FVM can simultaneously manage different types of complexities, i.e. heterogeneous formations, fractures, irregular boundaries, etc. Obviously, some previous knowledge of the reservoir is needed to set up a reliable reservoir model; however, if this is not available, the test interpretation is not feasible or misleading regardless of the approach that is used.

The results of the simulated well tests demonstrate the suitability and effectiveness of the FVM to describe the fluid flow even in complex scenarios; the potential of injection tests to identify reservoir boundaries will be the object of a future publication.

Finally, further work will include the use of the developed model for data assimilation so as to attempt the characterization of the reservoir heterogeneity (including faults) based on well test results. However, one of the limitations that cannot be overcome is that the information is not oriented, i.e. it is impossible to define in which direction a permeability variation or a flow barrier has been detected by the pressure disturbance. Another interesting development of the model would be inclusion of thermal effects so as to assess the impact of the temperature of the injected fluid (typically colder than the reservoir) on the pressure response.

\section{Acknowledgements}

The authors kindly acknowledge Schlumberger for providing the software Eclipse and Kappa Engineering for providing the software Saphir used in this research.

\section{References}

Abbaszadeh M and Kamal M. Automatic type-curve matching for well test analysis. SPE Formation Evaluation. 1988. 3(3): 567-577 (Paper SPE 16443). DOI: 10.2118/16443-PA

Amado L C and Pedrosa O A. A finite volume approach with triangular grids in reservoir simulation. SPE Advanced Technology Series. 1994. 2(1): 179-185. DOI: $10.2118 / 23633-P A$

Aziz K and Settari A. Petroleum Reservoir Simulation. 2002. Blitzprint Ltd. 476

Babu D K and Odeh A S. Productivity of a horizontal well. SPE Reservoir Engineering. 1992. 7(4): 454-455 (Paper SPE 25406)

Babu D K and Odeh A S. Authors' reply to discussion of productivity of a horizontal well. SPE Reservoir Engineering. 1992. 4(4): 454-455 (Paper SPE 25408)

Behie A. Comparison of nested factorization, constrained pressure residual, and incomplete factorization preconditionings. Paper SPE 13531 presented at the SPE Reservoir Simulation Symposium, Dallas, Texas, 10-13 Feb. 1985. DOI: 10.2118/13531-MS

Beretta E, Tiani A, Lo Presti G, et al. Value of injection testing as an alternative of conventional well testing: Field experience in a souroil reservoir. SPE Reservoir Evaluation \& Engineering. 2007. 10(2): 112-121 (Paper SPE 100283). DOI: 10.2118/100283-PA

Blazek J. Computational Fluid Dynamics: Principles and Applications. 2001. Oxford: Elsevier Science. 37-38

Bourdet D. Well Test Analysis: The Use of Advanced Interpretation Models. Elsevier Science B. V. The Netherlands. 2002. 426

Brigham W E. Discussion of productivity of a horizontal well. SPE Reservoir Engineering. 1990. 4(4): 254-255 (Paper SPE 20394)

Chen Z, Huan G and Ma Y. Computational Methods for Multiphase
Flows in Porous Media. SIAM. 2006. 531

Dickstein F, Lara A Q, Neri C and Peres A M. Modeling and simulation of horizontal wellbore-reservoir flow equations. Paper SPE 39064-MS presented at Latin American and Caribbean Petroleum Engineering Conference, Rio de Janeiro, Brazil, 1997. DOI: 10.2118/39064-MS

Eymard R, Gallou T and Herbin R. Finite Volume Methods. Handbook of Numerical Analysis. 1997. 7: 713-720

Faust C R and Mercer J W. An analysis of finite-difference and finiteelement techniques for geothermal reservoir simulation. Paper SPE 5742-MS presented at SPE Symposium on Numerical Simulation of Reservoir Performance, Los Angeles (CA), 19-20 Feb. 1976. DOI: 10.2118/5742-MS

Gunawan R, Nai-Shyong Y and Bennett C O. Application of injection tests for reservoir appraisals: A conceptual study. Paper SPE 77454-MS presented at the SPE Annual Technical Conference and Exhibition, San Antonio, Texas, 29 Sep. - 2 Oct. 2002. DOI: 10.2118/77454-MS

Hajibeygi H, Deb R and Jenny P. Multiscale finite volume method for non-conforming coarse grids arising from faulted porous media. Paper SPE 142205 presented at the SPE Reservoir Simulation Symposium, The Woodlands, Texas, USA, 21-23 Feb. 2011. DOI: 10.2118/142205-MS

Joshi S. Horizontal Well Technology. PennWell Books. Tulsa Oklahoma. USA. 1991. 165-201

Kelly R T. Non-uniqueness in well test analysis: Case studies. Paper SPE 36528 presented at the SPE Annual Technical Conference and Exhibition, Denver, Colorado, 6-9 Oct. 1996. DOI: 10.2118/36528MS

Klie H, Rame M and Wheeler M. Two-stage Preconditioners for Inexact Newton Methods in Multi-phase Reservoir Simulation. Center for Research on Parallel Computation, Houston. 1996. 38

Kuchuk F J, Goode P A, Wilkinson D J, et al. Pressure-transient behavior of horizontal wells with and without gas cap or aquifer. SPE Formation Evaluation. 1991. 6(1): 86-94 (Paper SPE 17413)

Kuchuk F J. Well testing and interpretation for horizontal wells. Journal of Petroleum Technology. 1995. 47(1): 36-41 (Paper SPE 25232). DOI: $10.2118 / 25232-\mathrm{PA}$

Lemonnier P A. Improvement of reservoir simulation by a triangular discontinuous finite element method. Paper SPE 8249 presented at the SPE Annual Technical Conference and Exhibition, Las Vegas, US, 23-26 Sep. 1979. DOI: 10.2118/8249

Leveque R J. Finite Volume Methods for Hyperbolic Problems. Cambridge University Press. 2002

Levitan M M. Application of water injection/falloff tests for reservoir appraisal: New analytical solution method for two-phase variable rate problems. Paper SPE 77532 presented at the SPE Annual Technical Conference and Exhibition, San Antonio, Texas, 29 Sep. 2 Oct. 2002. DOI: 10.2118/77532-MS

Mattax C and Dalton R. Reservoir Simulation. Monograph Series, SPE. Richardson, Texas. 1990. 17: 1-2

Mishev I and Jiang L. Mixed multiscale finite volume method for reservoir simulation in porous media with non local features. Paper SPE 141289 presented at the SPE Reservoir Simulation Symposium, The Woodlands, Texas, USA, 21-23 Feb. 2011. DOI: 10.2118/141289-MS

Nash S G and Sofer A. Linear and Nonlinear Programming. McGrawHill. 1996. 2: 37-43

Ozkan E and Raghavan R. Horizontal-well pressure analysis. SPE Formation Evaluation.1989. 4(4): 567-575 (Paper SPE 16378). DOI: $10.2118 / 16378$

Ozkan E and Raghavan R. New solutions for well-test-analysis problems: Part 2 - Computational considerations and applications. SPE Formation Evaluation. 1991. 6(3): 369-378 (Paper SPE 18616). 
DOI: 10.2118/18616-PA

Peaceman D W. Interpretation of well-block pressures in numerical reservoir simulation. SPE Journal. 1978. 18(3): 183-194 (paper SPE 6893). DOI: $10.2118 / 6893$

Peaceman D W. Discussion of productivity of a horizontal well. SPE Reservoir Engineering. 1990. 4(4): 252-253 (Paper SPE 20306)

Rozon B J. A generalized finite volume discretization method for reservoir simulation. Paper SPE 18414 presented at the SPE Symposium on Reservoir Simulation, Houston, Texas, 6-8 Feb. 1989. DOI: $10.2118 / 18414-\mathrm{MS}$

Saad Y and Schultz M H. GMRES: A generalized minimal residual algorithm for solving nonsymmetric linear systems. SIAM Journal on Scientific and Statistical Computing. 1986. 7(3): 856-869

Saad Y. A flexible inner-outer preconditioned GMRES algorithm. SIAM Journal on Scientific Computing. 1993. 14: 461-461

Santos R L A, Pedrosa O A and Correa A C F. An efficient finite volume approach for modelling miscible displacement. Paper SPE 23691-MS presented at SPE Latin America Petroleum Engineering Conference, Caracas, Venezuela, 8-11 Mar. 1992. DOI: 10.2118/23691-MS

Soliman M Y, Azari M, Ansah J and Kabir C S. Design, interpretation and assessment of short-term pressure-transient tests. Paper SPE Paper 90837 presented at the SPE Annual Technical Conference and Exhibition, Houston, Texas, 26-29 Sep. 2004. DOI: 10.2118/90837MS

Soliman M Y, Azari M, Ansah J and Kabir C S. Review and application of short-term pressure transient testing of wells. Paper SPE 93560 presented at the 14th SPE Middle East Oil and Gas Show and Conference, Bahrain, 4-5 Mar. 2005. DOI: 10.2118/93560-MS

Suprunowicz R and Butler R M. Discussion of productivity of a horizontal well. SPE Reservoir Engineering. 1992. 7(4): 453-454 (Paper SPE 25295)
Tripaldi G, Beretta E, Bertolini C, et al. Injection testing: An innovative field application in Berkine Basin, Algeria. 9th Offshore Mediterranean Conference \& Exhibition, Ravenna, Italy, 25-27 Mar. 2009

Van der Vorst H A and Vuik C. GMRESR: A family of nested GMRES methods. Numerical Linear Algebra with Applications. 1994. 1(4): 369-386

Verga F and Rocca V. Green methodologies to test hydrocarbon reservoirs. American Journal of Environmental Sciences. 2010. 6(1): 1-10. DOI 10.3844/ajessp.2010.1.10

Verga F, Viberti D and Salina Borello E. A new 3-D numerical model to effectively simulate injection test. Paper SPE 113832 presented at the SPE Europec/EAGE Annual Conference and Exhibition, Rome, Italy, 9-12 Jun. 2008. DOI: 10.2118/113832-MS

Wan J, Durlofsky L J, Hughes T J R, et al. Stabilized finite element methods for coupled-geomechanics reservoir flow simulations. Paper SPE 79694 presented at the SPE Reservoir Simulation Symposium, Houston (TX), 3-5 Feb. 2003. DOI: 10.2118/79694MS

Welty D and Miller W. Automated history matching of well tests. Paper SPE 7695 presented at the SPE Reservoir Simulation Symposium. Denver, Colorado, 31 Jan. - 2 Feb. 1979. DOI: 10.2118/7695-MS

Xing $\mathrm{J}$ and Ma Y L. A new solution to ILU preconditioning parallelization and its applications in reservoir simulations. Paper SPE 35998-MS presented at Petroleum Computer Conference, Dallas, Texas, 2-5 June 1996. DOI: 10.2118/35998-MS

Zhan H. Analyticalstudy of capturetime to ahorizontalwell. Journal of Hydrology. 1999. 217(1-2): 46-54. DOI: 10.1016/S00221694(99)00013-X

(Edited Sun Yanhua) 